\title{
nature
protocols
}

\section{Author Correction: Structural prediction of protein models using distance restraints derived from cross-linking mass spectrometry data}

Zsuzsanna Orbán-Németh, Rebecca Beveridge, David M Hollenstein (D, Evelyn Rampler, Thomas Stranzl, Otto Hudecz, Johannes Doblmann, Peter Schlögelhofer and Karl Mechtler

Correction to: Nature Protocols https://doi.org/10.1038/nprot.2017.146, published online 8 February 2018.

In the version of this article initially published online, the authors used incorrectly defined restraints for specifying the distance between residues when using the HADDOCK portal. Following the publication of a Correspondence by the developers of the HADDOCK portal (Nat. Protoc. https://dx.doi.org/10.1038/s41596-018-0017-6, 2018) and a Reply by the authors of the Protocol (Nat. Protoc. https://dx.doi.org/10.1038/s41596-018-0018-5, 2018), the syntax in Step 21 has been corrected. In addition, the input files (available as Supplementary Data $5-7$ ) have been replaced.

Published online: 25 June 2018

https://doi.org/10.1038/s41596-018-0024-7 